\title{
Limited regeneration but functional preservation of hematopoietic stem cells in the mice developing leukemia via constitutive expression of notch1
}

\author{
Xiaoxia $\mathrm{Hu}^{1,2}$, Hongmei Shen ${ }^{2}$, Hui $\mathrm{Yu}^{2}$, Feng Xu ${ }^{2}$, Jianmin Wang ${ }^{1}$, Tao Cheng ${ }^{3}$ \\ ${ }^{1}$ Department of Hematology, Changhai Hospital, Shanghai 200433, China; ${ }^{2}$ Department of Radiation Oncology, University of \\ Pittsburgh School of Medicine, Cancer Stem Cell Program, University of Pittsburgh Cancer Institute Pittsburgh, PA 15213, USA; \\ ${ }^{3}$ State Key Laboratory of Experimental Hematology, Institute of Hematology, Chinese Academy of Medical Sciences and Peking \\ Union Medical College, Tianjin 300020, China
}

Leukemia development is a complex process involving both intrinsic and extrinsic factors. While many environmental factors have been studied, the impact of leukemic environment on normal hematopoietic stem cell (HSC) and hematopoietic progenitor cell (HPC) has not been definitively investigated. In this study, we have formally addressed this important issue by examining the potential functional alterations of HSC and HPC in the mice bearing Notch1-induced T acute lymphoblastic leukemia (T-ALL). As expected, progressive hematopoietic suppression was observed in the leukemic mice as assessed by both frequency and absolute number of immunophenotypically-defined HSC and HPC, which was highly consistent with poorer clonal growth of the hematopoietic cells in vitro. Further cellular assays documented an accelerated phase of cell proliferation without significantly altered apoptosis upon the exposure of HPCs to the leukemic environment. To measure the long-term engraftment of HSCs from leukemic environment, equal numbers of the cells isolated from leukemic or control mice and competitor cells with distinct congenic markers were co-transplanted into new lethally irradiated recipients. Unexpectedly, the multilineage engraftment level of the hematopoietic cells isolated from the leukemic mice was significantly higher than that of the control group. Moreover, HSCs from leukemic environment remained functional in serial transplant recipients. Finally, to explore the underlying molecular mechanisms for the enhanced function of normal HSC in the competitive repopulation model, we examined a number of cell cycle and self-renewal regulators in HSC and HPC from leukemic marrow and control group at the time of harvest prior to transplantation by qRT-PCR. There was a significant decrease in p18 expression when compared with control, whereas p21 expression was significantly increased. Notch1, Gfi1 and c-myc signalings were also elevated in the HSCs from leukemic environment. In summary, our current study provides the first definitive evidence for the specific inhibition of normal HSC growth in the leukemic bone marrow. However, to our surprise, the HSCs isolated from the T-ALL hosts could better engraft in the recipients. Therefore, this study has important implications for HSC transplantation as well as leukemogenesis.

Keywords: leukemia environment, hematopoietic stem cell, proliferation, self-renew

Cell Research (2008) 18:s154. doi: 10.1038/cr.2008.244; published online 4 August 2008

Correspondence: Tao Cheng

E-mail: Chengt@upmc.edu 